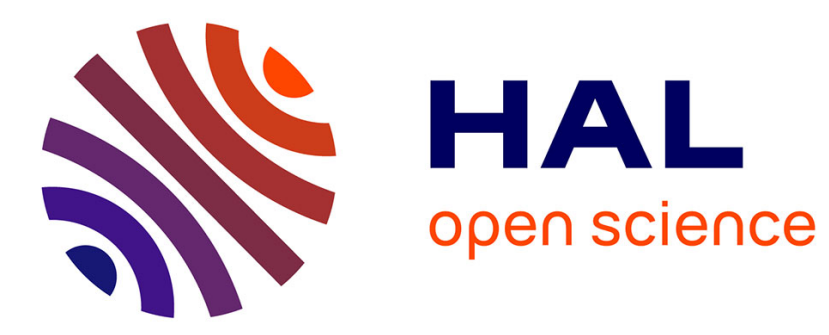

\title{
Les choix des conjoints en agriculture
}

Christophe Giraud, Jacques Rémy

\section{To cite this version:}

Christophe Giraud, Jacques Rémy. Les choix des conjoints en agriculture. Revue d'Etudes en Agriculture et Environnement - Review of agricultural and environmental studies, 2008, 88, pp.21-46. hal-01201184

\section{HAL Id: hal-01201184 \\ https://hal.science/hal-01201184}

Submitted on 17 Sep 2015

HAL is a multi-disciplinary open access archive for the deposit and dissemination of scientific research documents, whether they are published or not. The documents may come from teaching and research institutions in France or abroad, or from public or private research centers.
L'archive ouverte pluridisciplinaire HAL, est destinée au dépôt et à la diffusion de documents scientifiques de niveau recherche, publiés ou non, émanant des établissements d'enseignement et de recherche français ou étrangers, des laboratoires publics ou privés. 


\title{
Les choix des conjoints en agriculture
}

\author{
Christophe GIRAUD*, Jacques RÉMY** \\ * Université Paris Descartes, CERLIS (CNRS UMR 8070), 45 rue des Saints-Pères, 75270 Paris \\ cedex 06 \\ e-mail: christophe.giraud@parisdescartes.fr \\ ** INRA, UR1217 MONA, Ivry-sur-Seine, France
}

Résumé - Le monde agricole a longtemps été caractérisé par une relative fermeture sur lui-même. Fermeture professionnelle qui laisse peu de place aux nouveaux entrants d'origines sociales diverses, fermeture conjugale avec un choix relativement circonscrit aux filles d'agriculteur et restreint par la démographie des campagnes. La montée de l'hétérogamie, non seulement professionnelle (les femmes travaillent de plus en plus à l'extérieur de l'exploitation) mais sociale (les femmes sont de moins en moins issues du milieu agricole), remet en question cette image. Cette ouverture conjugale n'est pas uniforme : les agriculteurs des secteurs les plus spécialisés, comme la céréaliculture, sont plus enclins à avoir une conjointe extérieure à l'agriculture ; elle sera la partenaire dans une vie familiale qui ne se confondra pas avec celle de l'exploitation. Dans les secteurs moins spécialisés - comme les systèmes de polycultureélevage ou dans la production de lait avec un fort travail d'astreinte - ils ont plus tendance à avoir des conjointes filles d'agriculteurs qui travaillent avec eux, bref des collaboratrices dans une vie familiale centrée sur le travail.

Mots-clés: hétérogamie, ouverture sociale, agriculture, choix du conjoint, autonomie, activité professionnelle féminine

\section{Choosing a partner in agriculture}

Summary - The French agricultural world has long been characterized as being closed in on itself. This may take the form of a professional closure which leaves little place to new arrivals of diverse social origins, or the form of marital closure with relatively limited choices for the daughters of farmers given the demographic constraints of the countryside. The rise of heterogamy, not only professional (women work more and more outside the farm) but also social (women come less and less from the agricultural community), challenges this image. This marital opening is not uniform: farmers in the most specialized sectors such as grain cultivation are more inclined to be married to women unconnected to agriculture. These wives will be partners to a family life which does not merge with that of the farm. Farmers in less specialized sectors, like polycultural farming, raising livestock, or those with strong work obligations like milk production, are more likely to marry the daughters of farmers who go on to work with them - in other words, making them collaborators in a family life focused on work.

Keywords: heterogamy, social openness, agriculture, partner choice, autonomy, female employment 


\section{Introduction}

Dans le travail dont il est rendu compte ici, nous nous posons la question de la reproduction du «monde agricole " sous l'angle de la formation des couples. Girard (1980, pp. 188-192) constate que l'homogamie socioprofessionnelle est la plus forte chez les agriculteurs et dans les couches les plus favorisées de la population (mais que les premiers choisissent leur conjoint à proximité de leur lieu de résidence, alors que les seconds ont plus tendance à les choisir venant de lieux plus lointains). Comme le montre Desrosières (1978), cette fermeture conjugale n'a pas le même sens selon la position dans la hiérarchie sociale : choisie par les couches supérieures de la société, la fermeture est en revanche subie par les couches populaires les plus pauvres. Bourdieu a mis en évidence comment, dans le Béarn, même les aînés (les héritiers) de certaines familles paysannes ne trouvaient plus de conjointes: les positions relativement enviables de petits paysans propriétaires qu'ils occupaient encore avant-guerre se trouvaient dévalorisées aux yeux des jeunes filles qui leur ont préféré des jeunes, urbains ou ruraux, appartenant à d'autres catégories sociales (Bourdieu, 1962, 1972, 1989a). Les paysans seraient donc condamnés soit à la non-reproduction physique et sociale (Champagne, 2002), soit à la clôture sur soi afin d'assurer la reproduction d'une partie du groupe ${ }^{1}$.

Comment le groupe socioprofessionnel des agriculteurs - quarante ans après « $L a$ fin des paysans » (Mendras, 1967) - recrute-t-il aujourd'hui ses nouveaux membres? Comment se mélange-t-il avec les autres groupes socioprofessionnels ? L'ouverture ou la fermeture sociale relative du groupe des agriculteurs est évaluée ici à travers le choix du conjoint, porteur de capitaux spécifiques, et de la place qui lui est faite dans l'exploitation, c'est-à-dire le cloisonnement ou au contraire l'intégration de celui-ci dans le travail quotidien. Nous montrerons combien ce "groupe » agricole est divisé sur l'origine sociale et le statut professionnel des conjointes et que ces formes d'association renvoient à deux modes distincts de reproduction, l'un passant par l'intégration au collectif de travail actif sur l'exploitation, l'autre par l'indépendance des activités professionnelles dans le ménage comme c'est déjà le cas dans le monde salarié.

Les alliances entre les agriculteurs et les autres groupes sociaux peuvent être observées grâce à l'enquête «Emploi » de l'INSEE 2. L'enquête Emploi approche le groupe des agriculteurs à partir de la nomenclature des professions et catégories socioprofessionnelles (PCS) qui distingue trois niveaux: petits agriculteurs, agriculteurs moyens, gros agriculteurs, typologie dont l'adoption a rencontré une forte résistance (Desrosières et Thévenot, 1996, pp. 73-74). Une caractéristique centrale de ce milieu

\footnotetext{
${ }^{1}$ Les agriculteurs, selon Girard (1980) et Bozon et Héran (2006), ont plus tendance que les autres à trouver les conjoints dans des lieux ouverts, alors que plus on monte dans l'échelle sociale et plus les lieux où l'on rencontre son conjoint sont des lieux fermés ou privés. Les milieux favorisés se protègent des «mauvaises rencontres». On pourrait dire alors que les petits agriculteurs et autres membres de classe populaire se retrouvent souvent «enfermés dehors ».

${ }^{2}$ Nous remercions le Centre Quételet qui a mis à notre disposition l'enquête Emploi de 2000 (EE2000) et de 1990 (EE1990) et notamment Alexandre Kych pour sa disponibilité. Nous sommes bien sûr seuls responsables des traitements statistiques. Nous remercions également les deux lecteurs de la revue de leurs fort judicieuses observations.
} 
est, en effet, d'être fortement travaillé par l'image (le mythe) de l'unité agricole construite par une partie de la profession (Coulomb et al., 1990). En traitant les agriculteurs comme un groupe social nous n'entendons pas valider cette construction idéologique, mais plutôt bâtir provisoirement un outil d'analyse qui permette des comparaisons statistiquement pertinentes entre les différents groupes socioprofessionnels. Au cours de l'analyse nous serons amenés à nuancer notre construction provisoire de ce monde de l'agriculture.

L'enquête Emploi porte sur les ménages et comporte des informations individuelles sur tous leurs membres de plus de 15 ans. Depuis 1982, elle donne des renseignements sur le père de la personne de référence du ménage ainsi que sur le père de son conjoint : ces renseignements nous serviront d'indicateurs respectivement de l'origine sociale du conjoint et de celle de sa compagne. Il s'agit là d'un choix contraint par les informations disponibles dans cette base. Celle-ci tombe sous le coup des critiques faites par Delphy (1977) qui relevait déjà le biais sexiste des constructions statistiques réduisant la position sociale des ménages à la profession du seul « chef de ménage ». Une analyse plus soucieuse du rôle des femmes devrait aussi prendre en compte systématiquement la profession de la mère (pour définir la position sociale d'un ménage ou l'origine sociale d'un individu). A la différence de l'enquête FQP (formation et qualification professionnelles) de 1993, l'enquête Emploi ne donne pas d'indication sur la profession de la belle-mère de l'agriculteur. Elle ne renseigne pas non plus sur l'éventuelle double activité des pères et beaux-pères : or, on peut penser qu'en milieu rural une partie des individus interrogés ont souvent une activité agricole, que celle-ci constitue la profession principale ou un «travail à côté » (Weber, 1989). Lorsque nous parlons de «l'origine sociale de la conjointe », il s'agit donc de la profession principale du père de celle-ci. De plus, lorsque nous parlerons de filles d'origine agricole, nous désignerons les femmes dont le père est agriculteur ou ouvrier agricole. Dans la perspective de la présente étude, la socialisation dans un milieu professionnel parâtt plus centrale pour les comparaisons à venir que la position particulière du père dans ce milieu. Enfin, l'enquête Emploi approche les couples, qu'ils soient mariés ou non. Nous utiliserons donc les termes «conjoint» ou « conjointe».

Nous examinerons d'abord la question de l'évolution de l'homogamie en milieu agricole. Avec le développement du travail professionnel des femmes mariées, l'attention des analystes (notamment Vallet, 1986) s'est plutôt portée sur l'homogamie socioprofessionnelle, c'est-à-dire la propension à s'unir au sein du même milieu socioprofessionnel (identifié par la PCS). Aujourd'hui, d'autres auteurs (comme Bozon et Héran, 2006 ; Vanderschelden, 2006) insistent sur l'homogamie culturelle. Notre propos se centre, dans la partie suivante, sur la question de l'homogamie sociale, c'està-dire de la propension à s'unir au sein du même milieu d'origine. L'ascendance sociale des conjointes nous paraît en effet un indicateur central des dispositions dont elles sont porteuses et qui conditionnent leurs choix, notamment en matière d'activité professionnelle. Nous évoquons ensuite moins l'homogamie professionnelle que le poids de l'ascendance sociale des conjointes sur la manière dont elles s'intègrent à l'exploitation agricole du mari. L'ultime point aborde la question des conséquences, sur 
la vie conjugale et le rapport à l'exploitation, de l'ouverture à des conjointes d'autres origines et d'autres milieux.

Si l'ouverture progressive à d'autres milieux sociaux semble un fait d'évidence dans le milieu agricole, au-delà des effets mécaniques de la démographie, nous nous poserons la question du sens et des limites de cette ouverture. L'ouverture est-elle synonyme d'intégration professionnelle des conjointes? Quels sont les métiers de l'agriculture les plus ouverts conjugalement ? Deux populations différentes seront à cet égard distinguées.

\section{L'ouverture progressive du champ des possibles conjugaux}

Dans cette première partie, nous allons examiner les transformations récentes des choix conjugaux des agriculteurs et tenter d'en mesurer l'ampleur et les effets sur la composition de ce groupe socioprofessionnel. Ces évolutions peuvent-elles être lues comme le signe d'une plus grande ouverture du groupe des agriculteurs à d'autres groupes sociaux?

\subsection{Une homogamie sociale agricole encore importante mais une hétérogamie en forte hausse}

Nous souhaitons montrer ici que les agriculteurs exploitants ont élargi l'aire sociale de choix de leur conjointe, c'est-à-dire comment ils s'unissent désormais davantage avec des femmes venant d'autres milieux sociaux. Cette homogamie sociale est approchée de deux manières différentes: la première manière, la plus classique, suivie notamment par Alain Girard, est d'examiner le lien entre la profession du mari et celle de son beau-père. On calcule ainsi un taux de couples homogames. Cette manière de procéder reste cependant limitée dans la mesure où elle compte les couples homogames sans prendre en compte la structure du marché matrimonial (c'est-à-dire le nombre d'agriculteurs et de filles d'agriculteurs disponibles pour une union). On mettra donc en rapport ce chiffre avec le taux de filles d'agriculteur en couple. Girard (1980) montre ainsi, qu'en 1959, $83 \%$ des agriculteurs avaient épousé des filles d'agriculteur ou d'ouvrier agricole alors qu'elles constituaient 34,5\% des femmes mariées. L'homogamie en agriculture est donc presque trois fois plus fréquente que si le choix du conjoint se faisait au hasard (le rapport entre les deux taux, également appelé indice d'homogamie $^{3}$, est de 2,4). Quarante ans plus tard, en 1990, l'homogamie mesurée à

${ }^{3}$ L'indice d'homogamie est ici calculé de manière peu usuelle comme le rapport entre deux taux $\left(n_{i j} / n_{i}\right) /\left(n_{. j} / n\right)$, où $n_{i j}$ est le nombre de couples où l'homme est de la PCS $i$ et la conjointe de l'origine sociale $j ; n_{i}$. est le nombre de couples où le mari fait partie de la PCS $i ; n_{. j}$ est le nombre de couples dont la conjointe a l'origine sociale $j$; enfin $n$ est le nombre total de couples. Il est généralement défini comme le rapport entre la proportion de couples homogames observée $\left(\mathrm{n}_{\mathrm{ij}} / \mathrm{n}\right)$ et la proportion que l'on aurait dû obtenir si les unions s'étaient faites au hasard, proportion calculée à partir de la formule des probabilités indépendantes soit $\left(\mathrm{n}_{\mathrm{i}} / \mathrm{n}\right) *\left(\mathrm{n}_{\mathrm{j}} / \mathrm{n}\right)(c f$. Vanderschelden, 2006, p. 39). L'indice d'homogamie s'écrit alors dans les deux cas $I=\left(n * n_{i j}\right) /\left(n_{i} . * n_{. j}\right)$. L'indice d'homogamie présente cependant des biais quand il s'agit de faire les comparaisons entre plusieurs dates, notamment lorsque la distribution socioprofessionnelle des femmes a tendance à s'écarter de celle des hommes. Nous resterons donc prudents sur les évolutions. Pour d'autres techniques comme les régressions log-linéaires qui permettent de mieux approcher ces évolutions, voir Vallet (1986) ou Vanderschelden (2006). 
partir du même indicateur ${ }^{4}$ semble avoir reculée : 70,4\% des agriculteurs seulement avaient une conjointe d'origine agricole (y compris fille de salarié agricole). Mais les filles d'agriculteur ne représentent plus que $24,2 \%$ des conjointes dans toute la population. Les unions homogames sont donc trois plus fréquentes dans la réalité que si le choix du conjoint se faisait au hasard. L'indice d'homogamie de 2,9 en 1990 montre même un accroissement de la propension à s'unir avec une conjointe issue du même milieu. Enfin en 2000, on peut constater un recul de l'homogamie par rapport à 1990 au vu des unions observées (EE 2000), 55,0\% des agriculteurs ont une conjointe issue du milieu agricole ${ }^{5}$ alors que 20,7 \% des hommes seulement dans l'ensemble de la population se mettent en couple avec des filles d'agriculteur (ou de salarié agricole). L'indice d'homogamie est de 2,6 6 .

D'après ces chiffres, l'évolution de l'homogamie n'est en rien linéaire. Si la part des couples homogames baisse régulièrement, l'indice d'homogamie baisse depuis 1990, mais reste pourtant plus élevé que celui de 1959. Une forte tendance à l'homogamie caractérise donc toujours le milieu agricole. L'homogamie d'aujourd'hui ne ressemble pourtant pas à celle d'hier. Dans la situation démographique actuelle du groupe agricole, les unions hétérogames sont aujourd'hui aussi nombreuses que les unions homogames alors qu'elles étaient très rares il y a cinquante ans au moment de l'enquête de Girard. L'hétérogamie est devenue une réalité très fréquente et non plus exceptionnelle.

Cette ouverture récente des agriculteurs aux conjointes issues d'autres catégories socioprofessionnelles se confirme quand on l'examine sous un deuxième angle, celui des générations ${ }^{7}$. Si l'évolution de l'homogamie est sensible entre 1959 et 2000 quand on la calcule sur le stock global des couples, on peut également capter son évolution en s'intéressant au taux d'homogamie par classe d'âge d'un des conjoints (ici la femme) à une date donnée. Afin de préserver des effectifs conséquents, l'homogamie sociale est évaluée sur la base de la définition de Girard (profession du mari identique à celle de

\footnotetext{
${ }^{4}$ En 1959, Girard s'appuie sur les seuls couples mariés. «Au début des années 60, $15 \%$ des unions se formaient hors mariage (...). Cette proportion atteignait $20 \%$ en 1970 et $87 \%$ en $1990 »$ (INSEE, 1995, p. 52). Cette situation de concubinage a de surcroît de plus en plus tendance à durer. Aujourd'hui donc, le concubinage ne peut plus être ignoré et doit être pris en compte pour une étude sur le choix du conjoint. Nous effectuons donc nos calculs sur la base des couples cohabitants, mariés ou non.

5 C'est-à-dire y compris filles de salarié agricole.

${ }^{6}$ En réalité, cette manière de mesurer l'homogamie est devenue insatisfaisante aujourd'hui : à la différence de 1959, les agriculteurs de l'an 2000 sont 14,4\% à ne pas avoir un père agriculteur. L'union de ceux-ci avec une conjointe d'origine agricole devrait alors être codée comme hétérogame et non pas homogame. Bref, il faudrait pour la population des agriculteurs masculins prendre en compte l'origine sociale des deux conjoints. Les indices d'homogamie rénovés sont alors de 2,9 en 1990 et de 2,4 en 2000. L'interprétation des évolutions de cet indice reste la même.

7 Une troisième démarche aurait pu consister en un suivi des cohortes des nouveaux couples observés dans chaque enquête Emploi et une analyse du taux d'homogamie parmi ces cohortes. Si ce suivi est sans doute le plus rigoureux, les effectifs annuels des nouvelles unions avec des exploitants agricoles masculins sont relativement réduits dans l'enquête Emploi. L'approche par " génération " n'est pas un suivi de cohortes à proprement parler car le stock de couples observé pour une classe d'âge donnée en 2000 contient de nouveaux couples non enregistrés en 1990 et ne permet pas de mesurer le nombre de couples existant en 1990 mais qui ont rompu entre-temps.
} 
son beau-père). En 2000, si en moyenne 54,8\% des conjointes d'agriculteurs sont d'origine agricole (y compris de salariés agricoles), ce sont 38,8\% des conjointes de 25 à 30 ans et 70,7\% des conjointes de 60 à 65 ans qui sont d'origine agricole. Ce taux crôit linéairement en fonction de l'âge de la conjointe : plus les femmes d'agriculteurs sont âgées et plus elles ont tendance à être d'origine agricole. Au-dessous de 40 ans, les conjointes d'agriculteurs filles d'agriculteurs (ou de salariés agricoles) sont désormais minoritaires par rapport à celles qui ont une autre origine sociale.

Les données de 1990 montrent la même tendance à une ouverture du champ des possibles, mais les taux de recrutement homogame sont globalement plus élevés qu'en 2000 : si en moyenne 69,3\% des conjointes sont d'origine agricole (y compris enfants de salariés agricoles), on trouve seulement $47,1 \%$ des conjointes de 25 à 30 ans et tout de même 76,9\% des conjointes de 60 à 65 ans. Le même mouvement de croissance linéaire du taux de conjointes homogames en fonction de l'âge est constatable. Cependant l'indice d'homogamie pour chaque classe d'âge n'a pas reculé. Il se maintient à un niveau élevé, y compris pour les classes d'âge jeunes ${ }^{8}$. On peut donc retrouver la conclusion faite précédemment: l'homogamie sociale reste une tendance forte du groupe agricole, mais la part des conjointes d'origine agricole et donc des couples homogames tend à reculer et devient minoritaire dans les classes d'âge les plus jeunes. Le recul du taux des unions homogames ne traduit donc pas une moindre propension à se marier dans le même milieu mais plutôt, du côté des agriculteurs, une adaptation au plus faible nombre de filles d'agriculteurs disponibles sur le marché matrimonial.

La réduction drastique de la taille du groupe agricole dans les cinquante dernières années explique en partie cette ouverture, car les hommes agriculteurs sont comparativement de moins en moins confrontés à des filles d'agriculteurs. Grâce à la croissance récente de la population rurale (la renaissance rurale selon Kayser, 1997), la distance géographique ne redouble plus la distance sociale entre les conjoints. En termes de probabilités, les chances d'épouser une femme d'origine non agricole augmentent mécaniquement. Nous retrouvons ici l'explication centrale donnée par Jégouzo (1991) de l'évolution de l'agriculture vers des unions moins homogames.

Toutefois, les chances théoriques ne suffisent jamais à expliquer les unions réelles : la perception de l'aire des possibles sociaux, les chances physiques de rencontre (même dans un milieu rural socialement plus diversifié qu'auparavant) sont des médiateurs puissants qui modifient le calcul abstrait des probabilités (Bozon et Héran, 2006). Outre la permanence d'une forte homogamie sociale, ces chiffres confirment aussi le fort accroissement, au cours du temps, de l'attractivité matrimoniale des hommes travaillant dans le monde agricole.

\footnotetext{
8 On peut noter qu'en dessous de 45 ans, en 1990 comme en 2000, plus les conjointes sont jeunes et plus l'indice d'homogamie semble élevé. Il s'agit sans doute moins d'un renforcement de la propension à s'allier avec une conjointe du même milieu dans les jeunes générations (puisque de 1990 à 2000, l'indice d'homogamie baisse fortement) que d'un rapport différencié des individus à la vie en couple : les couples homogames semblent, au vu de ces statistiques, se former plus tôt que les couples hétérogames. Nos données ne nous permettent cependant pas de vérifier précisément cette hypothèse.
} 
Tableau 1. Origine sociale de la conjointe en fonction de son âge quinquennal en 2000 et 1990

\begin{tabular}{lcccccc}
\hline $\begin{array}{c}\text { Classes d'âge } \\
\text { de la conjointe } \\
\text { en 2000 } \\
\text { et 1990 }\end{array}$ & $\begin{array}{c}\text { Filles } \\
\text { d'agriculteurs }\end{array}$ & $\begin{array}{c}\text { Filles } \\
\text { d'ouvriers }\end{array}$ & $\begin{array}{c}\text { Filles } \\
\text { d'une autre } \\
\text { origine }\end{array}$ & Total & $\begin{array}{c}\text { Part des filles } \\
\text { d'agriculteurs } \\
\text { dans la } \\
\text { population totale }\end{array}$ & $\begin{array}{c}\text { Indice } \\
\text { d'homogamie }\end{array}$ \\
\hline 20-25 ans & 41,2 & 35,3 & 23,5 & 100,0 & 6,4 & 6,4 \\
25-30 ans & 39,6 & 34,0 & 26,4 & 100,0 & 9,7 & 4,0 \\
& 38,8 & 36,3 & 25,0 & 100,0 & 7,9 & 4,9 \\
30-35 ans & 47,1 & 22,5 & 30,4 & 100,0 & 12,8 & 3,7 \\
& 39,4 & 32,9 & 27,7 & 100,0 & 10,9 & 3,6 \\
35-40 ans & 55,0 & 15,9 & 29,1 & 100,0 & 16,0 & 3,4 \\
& 46,4 & 24,0 & 29,6 & 100,0 & 13,0 & 3,6 \\
40-45 ans & 59,5 & 14,4 & 26,1 & 100,0 & 19,1 & 3,1 \\
& 50,5 & 26,8 & 22,7 & 100,0 & 17,3 & 2,9 \\
45-50 ans & 66,7 & 15,2 & 18,1 & 100,0 & 22,3 & 3,0 \\
& 52,0 & 24,0 & 24,0 & 100,0 & 19,9 & 2,6 \\
50-55 ans & 79,9 & 10,1 & 10,1 & 100,0 & 26,0 & 3,1 \\
& 74,9 & 11,3 & 13,9 & 100,0 & 23,7 & 3,1 \\
55-60 ans & 81,5 & 7,4 & 11,1 & 100,0 & 31,0 & 2,6 \\
& 67,5 & 7,8 & 24,7 & 100,0 & 26,6 & 2,5 \\
60-65 ans & 79,7 & 9,5 & 10,8 & 100,0 & 31,3 & 2,5 \\
& 70,7 & 12,2 & 17,1 & 100,0 & 30,0 & 2,4 \\
65-70 ans & 76,9 & 10,3 & 12,8 & 100,0 & 32,9 & 2,3 \\
& 75,0 & 20,8 & 4,2 & 100,0 & 34,2 & 2,2 \\
70 et plus & 94,6 & 5,4 & 0,0 & 100,0 & 35,2 & 2,7 \\
& 91,7 & 0,0 & 8,3 & 100,0 & 35,5 & 2,6 \\
\hline Total & 78,9 & 5,3 & 15,8 & 100,0 & 37,4 & 2,1 \\
\hline Effectifs & 54,8 & 22,6 & 22,6 & 100,0 & 16,1 & 3,4 \\
& 69,3 & 13,0 & 17,7 & 100,0 & 24,2 & 2,9 \\
\hline
\end{tabular}

Source et notes: EE2000, N = 1135 couples où l'homme est agriculteur à titre principal ou secondaire et 44345 couples avec conjoint de tous milieux sociaux. EE1990, $N=1722$ couples où l'bomme est agriculteur à titre principal ou secondaire et 40626 couples avec conjoint de tous milieux sociaux.

Lecture: En 2000, 41,2\% des conjointes d'agriculteurs âgées de 20 et 25 ans ont une origine agricole, contre seulement $39,6 \%$ en 1990. Les chiffres entre parenthèses sont des effectifs.

\subsection{L'ouverture et les stratégies matrimoniales}

Ni en 1990, ni en 2000, nous ne constatons de différences significatives dans les choix matrimoniaux des différentes strates d'agriculteurs ${ }^{9}$ : la dimension économique de l'exploitation ne semble pas jouer de rôle sur le choix du conjoint, mais des évolutions sont constatables d'une enquête à l'autre. En dix ans, c'est la part des conjointes d'origine ouvrière qui a le plus progressé (une conjointe d'agriculteur sur cinq en 2000

${ }_{9}$ Le test du Khi2 pour les années 1990 et 2000 ne nous permet pas de rejeter l'hypothèse d'indépendance. 
contre une sur huit en 1990), compensant la baisse de celle des conjointes d'origine agricole (une conjointe d'exploitant sur deux en 2000 contre deux sur trois, dix ans plus tôt) (cf. tableau 2).

Tableau 2. Dimension économique de l'exploitation et recrutement des conjointes en 2000 et 1990

\begin{tabular}{lcccc}
\hline & $\begin{array}{c}\text { Filles d'origine } \\
\text { agricole }\end{array}$ & $\begin{array}{c}\text { Filles d'origine } \\
\text { ouvrière }\end{array}$ & $\begin{array}{c}\text { Filles d'une } \\
\text { autre origine }\end{array}$ & Total \\
\hline \multirow{2}{*}{ Petites exploitations } & 51,2 & 24,8 & 24,0 & $100,0(129)$ \\
& 69,7 & 12,3 & 18,0 & $100,0(390)$ \\
Exploitations moyennes & 55,7 & 23,7 & 20,6 & $100,0(228)$ \\
& 68,7 & 13,9 & 17,4 & $100,0(539)$ \\
Grosses exploitations & 55,5 & 21,5 & 23,0 & $100,0(708)$ \\
& 72,1 & 11,8 & 16,1 & $100,0(671)$ \\
\hline \multirow{3}{*}{ Total (agriculteurs) } & $54,8(622)$ & $22,6(256)$ & $22,6(257)$ & $100,0(1135)$ \\
& $69,3(1194)$ & $13,0(224)$ & $17,7(304)$ & $100,0(1722)$ \\
\hline
\end{tabular}

Source et notes: EE2000, N = 1135 couples où l'homme est agriculteur à titre principal ou secondaire. EE1990, $N=1722$ couples où l'bomme est agriculteur à titre principal ou secondaire.

Lecture : 51,2\% des petits exploitants en 2000 (et 69,7\% en 1990) ont une conjointe d'origine agricole. Les chiffres entre parenthèses sont des effectifs.

De 1990 à 2000, l'ouverture matrimoniale en direction des milieux populaires non agricoles, milieux moyens et supérieurs s'accrôt, même si ces derniers restent toujours sous-représentés (les agriculteurs choisissent pour conjointe une fille de salarié de classe moyenne ou supérieure moins souvent que la moyenne). En revanche, les agriculteurs ne s'unissent pas plus souvent avec des conjointes d'origine indépendante en 2000 qu'en 1990.

Quand ils se marient en dehors du cercle agricole, les agriculteurs ont donc plus fortement tendance que la moyenne à vivre avec des filles d'ouvriers. Les probabilités de rencontre au sein du milieu local semblent jouer, les ouvriers étant surreprésentés en milieu rural par rapport aux autres groupes sociaux. Mais on peut penser que les probabilités ne sont pas seules à entrer en jeu ici et, tant qu'à considérer les stratégies, il conviendrait de les examiner des deux côtés. Si la force de travail et l'endurance physique supposées des filles d'ouvriers peuvent être considérées avec faveur par de jeunes agriculteurs, les détenteurs de capitaux peuvent exercer une certaine attraction pour ces jeunes filles qui en sont dépourvues. S'il est très difficile d'accéder de l'extérieur à la profession d'agriculteur ou d'agricultrice, en raison de l'importance des divers capitaux à mobiliser, il peut être plus aisé d'obtenir ce statut par le mariage.

L'unification du marché matrimonial mise en lumière par Bourdieu (1989a) est bien réelle puisque la valeur des agriculteurs peut se voir reconnue désormais au-delà des proximités sociales et géographiques traditionnelles. Être agriculteur condamne de moins en moins à se limiter aux conjointes du seul cercle agricole. Cette diversification ne se fait pas cependant selon la logique de la proximité des capitaux (être possesseur d'un capital foncier ne rapproche conjugalement pas plus d'individus porteurs d'un capital commercial), mais plutôt selon une logique de proximité géographique et 
Tableau 3. Origine sociale des conjointes dans les couples hétérogames dont le conjoint est agriculteur en 2000

\begin{tabular}{lcccc}
\hline & $\begin{array}{c}\text { Filles } \\
\text { d'indépendants }\end{array}$ & $\begin{array}{c}\text { Filles de salariés supérieurs } \\
\text { et moyens }\end{array}$ & $\begin{array}{c}\text { Filles } \\
\text { d'ouvriers }\end{array}$ & Total \\
\hline Petites exploitations & $15,9(10)$ & $33,3(21)$ & $50,8(32)$ & 100,0 \\
Exploitations moyennes & $18,8(19)$ & $27,7(28)$ & $53,5(54)$ & 100,0 \\
Grandes exploitations & $18,7(59)$ & $33,0(104)$ & $48,3(152)$ & 100,0 \\
\hline Total agri & $18,4(88)$ & $31,9(153)$ & $49,7(238)$ & 100,0 \\
\hline Total & $16,7(5860)$ & $38,0(13345)$ & $45,4(15947)$ & 100,0 \\
\hline
\end{tabular}

Source et note : EE2000, N = 44345 couples où l'homme est agriculteur à titre principal ou secondaire.

Lecture: En 2000, 15,9\% des petits exploitants (soit un effectif de 10 exploitants) ont une conjointe issue du monde indépendant (artisan, commerçant, profession libérale).

culturelle. Enfin, cette plus grande attractivité respecte la logique des distances sociales: les femmes d'origine supérieure ont moins tendance à s'unir avec un agriculteur que les femmes plus proches socialement.

L'attractivité conjugale des agriculteurs s'est accrue à mesure que le poids démographique du groupe agricole a décru. Cette réduction qui s'est faite surtout par la disparition des plus petits et des plus fragiles, comme le constatent Merllié et Prévot (1997, p. 43), a contribué à faire évoluer positivement le statut d'ensemble du groupe, par la professionnalisation des individus et par la concentration des moyens sur un nombre réduit d'exploitations. Cela a alors permis de réduire la distance aux autres groupes sociaux, de mieux profiter des nouvelles proximités géographiques avec des groupes sociaux différents, de mieux négocier les rencontres avec des femmes socialisées dans d'autres milieux sociaux et ayant déjà une profession qu'elles souhaitent continuer d'exercer.

\section{L'ouverture conjugale et l'intégration sociale de la conjointe}

L'ouverture du groupe agricole vers d'autres horizons conjugaux est donc avérée pour les membres actifs masculins de ce groupe. En reprenant les expressions de Bourdieu (1989a), si les agriculteurs «donnent leurs filles » aux autres catégories sociales, les autres groupes sociaux "offrent-ils» aujourd'hui plus facilement leurs filles non seulement aux agriculteurs, comme on vient de le voir, mais aussi à la profession agricole? Cela dépend de la manière dont ces nouveaux entrants sont intégrés sur l'exploitation. Se lier à un agriculteur, est-ce encore vraiment aujourd'hui épouser son métier? L'ouverture sociale par le mariage ou le concubinage est-elle synonyme d'intégration professionnelle et sociale?

Une vaste gamme de possibilités s'offre au groupe agricole pour l'intégration de ces conjointes venant d'un autre univers, gamme qui varie de l'intégration professionnelle prônée par le syndicalisme agricole dans les années soixante à l'indépendance totale entre les activités professionnelles des conjoints (sans oublier celle qui consiste à assigner à la compagne un statut exclusivement familial). On sait que les femmes travaillent de moins en moins sur l'exploitation (Barthez, 1993, pp. 474-475; 
Rattin, 1991, pp. 31-34 et 2002a, p. 19) ${ }^{10}$, est-ce un effet de leur origine plus souvent extérieure au monde agricole?

\subsection{L'ouverture et le cloisonnement professionnel}

Pour Delphy (1998), le mariage organise l'appropriation par le mari des ressources féminines, en premier lieu l'appropriation de sa force de travail. Les travaux de nombreux chercheurs (Barthez, 1982; Lagrave, 1987) ont montré combien le monde de l'agriculture organisait l'inégalité professionnelle statutaire entre les conjoints (l'homme, chef d'exploitation et la femme, aide familiale) et, par là, l'exploitation du travail gratuit des conjointes. On sait cependant que la situation des femmes en agriculture s'est considérablement transformée : les conjointes sont moins souvent aides familiales, elles travaillent aussi plus souvent à l'extérieur de l'exploitation, elles ont acquis une certaine indépendance professionnelle (Barthez, 1993). Dans l'enquête Emploi 2000 (EE2000), seulement 43,4\% des conjointes d'un chef d'exploitation masculin se déclarent agricultrices en activité, soit onze points de moins que dix ans plus tôt. Bref, le modèle de l'exploitation conjugale prôné par les lois d'orientation de 1960-1962 est en recul. Dans quelle mesure ce recul peut-il être lié à ce recrutement plus ouvert des conjointes? Ce lien est souvent avancé verbalement, mais jamais précisément démontré. Nous allons donc essayer de montrer comment l'origine sociale de la conjointe pèse sur son mode d'intégration à l'agriculture.

\subsubsection{La profession et le statut professionnel de la conjointe}

L'engagement professionnel et l'autonomie de la conjointe peuvent être évalués à partir de leur statut professionnel ${ }^{11}$. L'origine agricole favorise très fortement l'acquisition d'un statut d'agricultrice, mais aussi celui d'aide familiale alors qu'à l'inverse, les conjointes d'origine non agricole (ouvrière et autre) ont deux fois plus tendance que les autres à avoir un statut de salariée ${ }^{12}$ (cf. tableau 4).

En 2000, les conjointes d'origine moyenne ou supérieure ont presque deux fois plus tendance que celles venant du monde agricole à avoir un statut de salariée du public ou du privé. Le rapport était de trois dix ans plus tôt. Les disparités entre les conjointes d'ascendance agricole et les autres sont donc en train de se réduire : les premières sont en train de se rapprocher des secondes dans cette tendance à avoir une profession extérieure à

\footnotetext{
${ }_{10}$ En 2003, sur la population des exploitations dites professionnelles, $75 \%$ des épouses d'exploitants de moins de vingt-cinq ans n'ont aucune activité sur la ferme (Rattin, 2006, p. 2).

11 Notons qu'il s'agit du statut professionnel au moment de la réalisation des enquêtes Emploi 2000 et 1990 et non pas du statut professionnel au moment du mariage. Cette variable, ainsi que celle de la profession de l'épouse, font donc l'impasse sur la mobilité professionnelle et les périodes d'inactivité des femmes après la mise en couple.

12 Les effectifs de l'enquête Emploi ne nous permettent pas d'analyser finement la PCS des conjointes. Nous les regroupons donc en grandes catégories, différentes selon les tableaux. On sait toutefois, à partir du recensement agricole réalisé en 2000 (RA2000), que $51 \%$ des conjointes ne travaillant pas sur l'exploitation sont employées, et que $20 \%$ font partie des professions intermédiaires comme institutrices ou infirmières (Rattin, 2002b, p. 4).
} 
l'exploitation ${ }^{13}$. Symétriquement, en 1990, être issue du milieu agricole conduit deux fois plus souvent les conjointes à se déclarer aides familiales ou agricultrices qu'être issue d'un milieu moyen ou supérieur (en 2000, le faible nombre des compagnes ne nous permet pas de conclure sur ce point). L'origine moyenne et supérieure permet de mieux défendre un statut professionnel indépendant de celui de l'agriculteur.

Ces constatations peuvent être mises en relation avec le report des engagements matrimoniaux qui est perceptible aujourd'hui chez les jeunes adultes : le mariage se fait à un âge plus tardif et, souvent, les conjointes ont déjà un emploi. Cette différence donne à penser qu'entre les femmes d'origine agricole et les autres, ce sont les secondes qui ont plus tendance que les premières à ne pas quitter leur emploi quand elles se mettent en couple, c'est-à-dire à préserver de fait leur autonomie professionnelle par rapport à leur mari. Le calcul économique y incite également : on sait que les revenus extérieurs contribuent à financer les investissements consentis sur l'exploitation, ou du moins à assurer la charge de l'entretien de la famille, et constituent donc un facteur qui entre largement en ligne de compte pour l'acceptation des demandes d'emprunts auprès des banques (voir infra).

La vie en couple construit donc ici, en fonction de l'origine sociale de la conjointe, des cloisonnements au niveau de la vie professionnelle des ménages. Après la mise en couple, les conjointes d'origine non agricole cherchent à préserver une certaine autonomie professionnelle par rapport à l'exploitation, alors que celles d'ascendance agricole qui pouvaient éventuellement travailler avant le mariage ou le concubinage vont plus fréquemment se fondre dans la seule scène professionnelle de l'exploitation (avec des statuts divers) (Rémy, 1980). Ces deux profils constituent deux souspopulations qu'il est intéressant de distinguer au sein des couples en milieu agricole.

\subsubsection{L'intégration ou la mise à distance des conjointes}

L'intégration à l'exploitation peut également se lire dans le régime matrimonial ; celui de la communauté réduite aux acquêts constitue depuis 1966 le régime par défaut. Celui de la séparation de biens suppose la rédaction d'un contrat par le notaire et marque la construction d'une plus grande distance entre le monde du mari et celui de l'épouse. Régime souvent utilisé par les indépendants, il est considéré par ceux-ci comme une manière de protéger le patrimoine de l'un d'une éventuelle faillite de l'autre conjoint (Barthez et Laferrère, 1996). Fiche et al. (1993, p. 4) ont montré, à partir de l'enquête sur les structures foncières du SCEES de 1992, que $89 \%$ des couples d'exploitants agricoles avaient opté pour le régime de la communauté réduite aux acquêts contre seulement $10 \%$ qui avaient choisi la séparation des biens. Le choix très majoritaire est donc celui de la mise en commun une fois le mariage réalisé ${ }^{14}$.

\footnotetext{
13 L'homogamie professionnelle baisse chez les agriculteurs de 1990 à 2000, ce qui confirme une analyse des stocks de couples de 1962 à 1982 (Vallet, 1986) et une analyse de cohortes de couples formés de 1930 à 1990 (Vanderschelden, 2006, p. 45).

14 Mais pas du partage total : un troisième régime dit de « communauté universelle » qui consiste à mettre dans les biens communs tous ceux que les deux conjoints possèdent avant le mariage ou ont acquis individuellement par héritage n'est choisi que par $1 \%$ des couples.
} 
Tableau 4. Statut professionnel et origine sociale de la conjointe dans les exploitations agricoles en 2000 et 1990

\begin{tabular}{lcccc}
\hline & $\begin{array}{c}\text { Filles } \\
\text { d'origine } \\
\text { agricole }\end{array}$ & $\begin{array}{c}\text { Fille } \\
\text { d'origine } \\
\text { ouvrière }\end{array}$ & $\begin{array}{c}\text { Filles } \\
\text { d'autres } \\
\text { origines }\end{array}$ & Total \\
\hline Agricultrices & 27,8 & 16,4 & 14,6 & $22,5(207)$ \\
& 22,1 & 9,4 & 14,8 & $19,4(253)$ \\
Autres indépendantes & 1,3 & 2,1 & 5,0 & $2,3(21)$ \\
& 1,4 & 4,0 & 6,1 & $2,5(33)$ \\
Aides familiales & 37,1 & 26,2 & 22,7 & $31,7(291)$ \\
& 59,9 & 47,7 & 31,4 & $53,5(699)$ \\
Salariées (hors Etat, dont intérimaires & 22,8 & 38,0 & 38,9 & $29,5(271)$ \\
et apprentis) & 9,4 & 24,9 & 26,6 & $14,3(186)$ \\
Salariées de l'Etat (et collectivités territoriales, & 11,1 & 17,4 & 18,7 & $14,0(129)$ \\
dont contrat aidés et stagiaires) & 8,2 & 14,1 & 21,0 & $10,5(136)$ \\
\hline Total & $100,0(526)$ & $100,0(195)$ & $100,0(198)$ & $100,0(919)$ \\
& $100,0(929)$ & $100,0(149)$ & $100,0(229)$ & $100,0(1307)$ \\
\hline
\end{tabular}

Source et notes: EE2000, N = 919 couples où l'homme est agriculteur à titre principal ou secondaire. EE1990, $N=1307$ couples où l'bomme est agriculteur à titre principal ou secondaire.

Lecture : 27,8 \% des conjointes d'origine agricole ont un statut d'agricultrices. Les chiffres entre parenthèses sont des effectifs.

Mais le choix de la séparation des biens, même minoritaire, connaît des variations importantes. Il touche particulièrement les exploitants les plus jeunes. Un exploitant sur quatre de moins de 35 ans choisit le régime de la séparation contre un sur quinze de plus de 45 ans. Les choix en matière de régime matrimonial semblent évoluer rapidement ${ }^{15}$. La séparation des biens concerne les exploitations les plus importantes. De plus, le travail à l'extérieur de l'exploitation d'un des deux conjoints favorise l'adoption de ce type de contrat. C'est le cas de bien des conjointes qui ont un emploi à l'extérieur et qui peuvent mieux garantir leurs avoirs personnels en cas de séparation ou de faillite ${ }^{16}$. Il touche enfin davantage les conjointes d'origine non agricole qui n'apportent pas de biens fonciers à l'exploitation (ce qui permet de protéger le capital de l'exploitation acquis après le mariage en cas de séparation) (Fiche et al., 1993).

15 Pour que la comparaison soit tout à fait rigoureuse, il faudrait que les jeunes célibataires qui vivront avec une conjointe avant 35 ans fassent les mêmes choix en matière de régime matrimonial que leurs congénères déjà en couple.

16 D'autres arrangements, comme donner à l'exploitation agricole un statut de société (notamment sous la forme d'une EARL - entreprise agricole à responsabilité limitée - unipersonnelle), permettent également à la conjointe de protéger ses biens propres d'une éventuelle faillite ou à l'exploitant de protéger son exploitation des effets d'un divorce. Le statut d'EARL est également souvent choisi par des couples où les deux conjoints sont agriculteurs. Cette forme juridique permet en effet à la conjointe d'avoir un statut égal à celui de son compagnon exploitant; cependant, certaines de ces associations ne visent qu'à obtenir des avantages fiscaux et ne correspondent pas à une véritable activité sur l'exploitation de la co-exploitante en titre, ce qui ne facilite pas l'interprétation des données (la diversité des types de sociétés tend également à rendre les statuts plus opaques). 
Le concubinage est également une manière de mettre à distance la conjointe, de ne pas l'intégrer dans les arrangements et modifications patrimoniales de la famille: il équivaut au régime de séparation de biens, mais il témoigne d'un degré supplémentaire de distance et d'informalité dans le couple. Le taux de concubinage est moindre chez les agriculteurs que dans le reste de la population $(8,6 \%$ des agriculteurs en couple contre $11,0 \%$ des personnes de référence masculine en couple en 2000), mais il est en forte progression quand on le compare à 1990 (où ces même taux sont respectivement de $3,4 \%$ et $7,0 \%$ ). Souvent prélude à une union plus institutionnalisée, le concubinage touche plus souvent les couples les plus jeunes, mais si l'on considère la tranche d'âge de 40 à 49 ans, le concubinage s'établit alors à 5,1\%, contre 7,7\% en moyenne sur la population entière de la même tranche d'âge ${ }^{17}$.

La montée des unions en séparation de biens ou du concubinage dans les jeunes générations et dans les couples professionnellement hétérogames montre à nouveau combien ces unions entre individus qui n'ont pas le même métier ne sont pas synonymes d'intégration, mais au contraire s'effectuent avec la garantie d'importants cloisonnements et d'importantes protections des activités personnelles des deux conjoints. A l'inverse, les unions de conjoints professionnellement homogames élèvent moins de barrières juridiques entre les patrimoines et les activités des individus.

\subsection{L'articulation de l'activité des conjointes au fonctionnement de l'exploitation}

Le travail à l'extérieur des conjointes a été interprété comme une recherche d'autonomie professionnelle, mais il peut aussi être le signe de l'absence de besoin de main-d'œuvre familiale dans l'exploitation. L'enquête Emploi de l'INSEE nous permet d'approcher les divers métiers de l'agriculture, en regroupant les orientations technico-économiques (OTEX) de la statistique agricole en huit grandes orientations productives. Dans quelle mesure les besoins en termes de travail favorisent-ils tel ou tel profil de conjointe?

\subsubsection{Les besoins de main-d'euvre de l'exploitation}

Les orientations techniques où les actifs font le plus appel à la main-d'œuvre familiale sont bien connues par la statistique agricole: l'élevage de bovins (surtout l'élevage laitier), la polyculture-élevage et les élevages d'herbivores et de granivores peu intensifs sont fortement demandeurs. Les métiers des cultures permanentes, l'arboriculture et la viticulture (vin de qualité), ainsi que l'horticulture font plus facilement appel à des salariés, souvent saisonniers (Rattin, 1992, p. 47 et 49). Dans d'autres orientations comme les élevages d'ovins-caprins ou la grande culture, le travail du seul chef d'exploitation suffit souvent (id., p. 49). Bref, les productions qui sont les plus spécialisées sont aussi celles où la demande de main-d'œuvre familiale est moindre, alors qu'elle est plus souhaitée dans les systèmes de production peu spécialisés (polyculture-élevage) ou spécialisés mais avec un fort travail d'astreinte (lait). Comment

17 Le taux de concubinage est de 22,8\% pour les agriculteurs masculins de moins de 40 ans et de $2,1 \%$ pour les agriculteurs âgés de 50 à 59 ans contre respectivement $29,1 \%$ et $1,9 \%$ pour les conjoints masculins au sein de la population totale des mêmes tranches d'âge. 
les besoins de main-d'œuvre familiale sont-ils corrélés avec le recrutement social des conjointes?

Le choix des conjointes (résultats confirmés pour 1990) différencie clairement deux champs d'activité :

Les chefs des exploitations les plus spécialisées (maraîchage, viticulture et arboriculture, granivores) ou encore celles de polyculture ont une tendance plus marquée que ceux des systèmes peu spécialisés à choisir des conjointes d'origine non agricole et qui ne travaillent pas sur l'exploitation. Faisant plus souvent appel au travail salarié, ces producteurs peuvent aussi mieux s'accommoder d'un travail à l'extérieur des conjointes, voire l'encourager.

Les exploitants les moins spécialisés (polyculture-élevage, élevage d'herbivores et de granivores) et ceux qui connaissent une forte part de travail d'astreinte (lait en particulier) convolent le plus souvent «en interne» et les conjointes demeurent sur l'exploitation. C'est là aussi qu'on a le plus tendance à accueillir des conjointes d'origine non agricole qui vont cependant rester sur l'exploitation. Ce sont des collaboratrices qui sont recherchées, à la différence de leurs collègues des autres orientations productives qui choisissent des conjointes ayant tendance à conserver leur autonomie professionnelle. Pour les premières, l'exploitation devient d'autant plus facilement cadre de vie et de travail qu'elles ont déjà connu cet univers agricole, qu'elles y ont été socialisées.

Tableau 5. Orientation productive de l'exploitation et profil socioprofessionnel des conjointes en 2000

\begin{tabular}{|c|c|c|c|c|c|c|}
\hline & $\begin{array}{c}\text { Conjointes } \\
\text { d'origine } \\
\text { agricole } \\
\text { et } \\
\text { agricultrices }\end{array}$ & $\begin{array}{l}\text { Conjointes } \\
\text { d'origine } \\
\text { agricole } \\
\text { et non } \\
\text { agricultrices }\end{array}$ & $\begin{array}{c}\text { Conjointes } \\
\text { d'origine } \\
\text { non agricole } \\
\text { et } \\
\text { agricultrices }\end{array}$ & $\begin{array}{l}\text { Conjointes } \\
\text { d'origine } \\
\text { non agricole } \\
\text { et non } \\
\text { agricultrices }\end{array}$ & $\begin{array}{l}\text { Conjointes } \\
\text { inactives }\end{array}$ & Total \\
\hline $\begin{array}{l}\text { Polyculture (culture } \\
\text { de terres labourables) }\end{array}$ & 25,5 & 25,0 & 11,2 & 34,6 & 38,3 & $\begin{array}{l}100,0 \\
(188)\end{array}$ \\
\hline $\begin{array}{l}\text { Systèmes spécialisés } \\
\text { (Maraîchage, } \\
\text { horticulture, viticulture, } \\
\text { arboriculture, } \\
\text { granivores) }\end{array}$ & 28,2 & 23,0 & 12,1 & 35,1 & 1,7 & $\begin{array}{l}100,0 \\
(174)\end{array}$ \\
\hline $\begin{array}{l}\text { Elevage d'herbivores } \\
\text { (bovins, ovins, ...) }\end{array}$ & 36,2 & 16,3 & 17,0 & 27,9 & 12,7 & $\begin{array}{l}100,0 \\
(276)\end{array}$ \\
\hline $\begin{array}{l}\text { Systèmes peu spécialisés } \\
\text { (polyculture-élevage, } \\
\text { élevage ovins caprins, } \\
\text { autres). }\end{array}$ & 36,4 & 23,5 & 16,8 & 22,2 & 1,1 & $\begin{array}{l}100,0 \\
(374)\end{array}$ \\
\hline Total & $30,1(333)$ & $19,9(220)$ & $13,8(152)$ & 25,9 (286) & $10,3(114)$ & $\begin{array}{l}100,0 \\
(1012)\end{array}$ \\
\hline
\end{tabular}

Source et note: EE2000, N = 1012 couples où l'homme est agriculteur à titre principal ou secondaire.

Lecture : $25,5 \%$ des exploitants en polyculture ont une conjointe d'origine agricole et qui travaille sur l'exploitation. Les chiffres entre parenthèses sont des effectifs. 
Le choix du conjoint s'appuierait ici sur un double mécanisme: une attractivité différenciée des hommes agriculteurs, fonction de l'autonomie et de la protection dont pourront jouir leurs futures conjointes en matière professionnelle; un besoin différencié de force de travail familiale lié au système de production. La diversité des dispositions à s'engager dans le collectif de travail ou à préserver son autonomie professionnelle est ici centrale. De ce point de vue, toutes les origines sociales ne se valent pas: les conjointes qui ont été éduquées dans la classe moyenne ou supérieure sont aussi les plus sensibles à la logique individualiste ambiante (Singly, 2000) et peuvent essayer de préserver davantage que les autres leur indépendance professionnelle en cloisonnant vie professionnelle, vie familiale et vie de l'exploitation. Ce sont aussi celles qui conviennent le moins aux collectifs de travail fondés sur la main-d'œuvre familiale. Elles sont en revanche présentes lorsque les exploitations fonctionnent avec un seul emploi familial (polyculture), ou avec des salariés (arboriculture, maraîchage...). Les conjointes d'origine agricole ont quant à elles plus tendance à s'intégrer dans l'équipe familiale de production et à officier sur des exploitations qui ont besoin avant tout de collaboratrices (exploitations en lait, en polyculture-élevage). L'origine sociale est donc fondamentale pour comprendre ce qui se joue dans le choix du conjoint.

L'ouverture conjugale des agriculteurs ne doit pas être vue comme uniforme : des exploitants plutôt peu spécialisés ou avec un fort travail d'astreinte, qui recherchent plutôt des conjointes d'origine agricole en lesquelles ils voient aussi des collaboratrices ${ }^{18}$, doivent être distingués d'un autre ensemble de producteurs (très spécialisés et faisant appel à de la main-d'œuvre salariée), plus sensibles aux jeunes filles venant d'autres groupes sociaux dont ils partagent la vie sans qu'elles s'intègrent à celle de l'exploitation. Ces deux mondes de l'agriculture sont très différents en termes de métiers et de position dans le groupe socioprofessionnel agricole et montrent une première source d'hétérogénéité dans ce qu'on avait jusqu’à présent considéré comme un ensemble homogène.

\subsubsection{Le travail à l'extérieur de la conjointe: une source alternative de revenu?}

L'ouverture matrimoniale croissante des agriculteurs semble donc varier sensiblement en fonction des attentes en matière de main-d'œuvre familiale. Mais les besoins de l'exploitation ne se limitent pas à la main-d'œuvre : la conjointe salariée à l'extérieur de l'exploitation peut aider en prenant en charge par son salaire une bonne partie des dépenses du ménage, elle peut contribuer également à financer l'exploitation en cas de « coup dur » et représenter une garantie pour les emprunts bancaires (Bessière, 2004 ;

\footnotetext{
18 Rattin (2002a) montre également, à partir des données du RA 2000, que s'oppose une population de jeunes conjointes qui délaissent le travail sur l'exploitation pour une activité professionnelle à l'extérieur (ce qui n'empêche pas les aides ponctuelles sur l'exploitation) à une autre population jeune qui travaille sur l'exploitation et y travaille de plus en plus en se «professionnalisant », c'est-à-dire en acquérant le statut de coexploitante ou en travaillant plus souvent à temps plein sur l'exploitation. Les premières se trouvent plutôt dans des régions de grandes cultures du centre de la France alors que les secondes officient plutôt dans les secteurs de l'élevage (hors-sol, ovins-caprins et viticulture). Le champ des données utilisées est toutefois différent du nôtre car Rattin prend aussi en compte les exploitations dont le chef est une femme.
} 
Delame et Thomas, 2007). Dans ce cadre, on peut s'attendre à ce que les petits exploitants, quand ils trouvent une conjointe, favorisent son travail à l'extérieur ${ }^{19}$. C'est cette idée couramment répétée d'un travail extérieur des conjointes en tant que stratégie alternative de reproduction des exploitations que nous allons tester.

En fait, contrairement à cette hypothèse, en 1990 et surtout en 2000, les agriculteurs des exploitations les plus grandes ont eu plus tendance que ceux des petites exploitations à s'engager avec des conjointes qui travaillent à l'extérieur de l'exploitation ${ }^{20}$ ( $c f$. tableau 6 ). Nous retrouvons ici la distinction que nous avons faite antérieurement entre deux segments du monde agricole: les exploitations les plus petites sont plus intégratives en termes professionnels pour les conjointes (même si cela se traduit par une absence de statut professionnel individuel ${ }^{21}$ ), alors que les plus importantes et souvent plus capitalistiques sont aussi celles où le cloisonnement professionnel et l'indépendance économique des conjointes sont les plus marqués.

Pour le premier groupe, il y a une mobilisation perceptible autour de l'exploitation et de la famille - parfois encore confondues - qui met en œuvre certaines valeurs et normes - un ethos - auxquelles les conjointes d'origine agricole adhèrent pour les avoir vécues dans leur propre famille (Rémy, 1977). Pour le second groupe, l'élargissement de l'aire (géographique et sociale) de rencontre de la conjointe se fait alors au prix d'un important travail de cloisonnement et de protection de celle-ci par rapport à l'exploitation et d'un abandon, par une fraction importante des exploitants familiaux qui y adhéraient profondément à la génération précédente, du modèle du couple exploitant des lois d'orientation des années 60 : ces conjointes s'assurent - ou se voient assurées - de la préservation d'une véritable autonomie personnelle qui s'articule avec les besoins de l'exploitation et du ménage (Bessière, 2004 et 2006).

Le groupe agricole est donc fortement clivé du point de vue de l'alliance. Dans certains métiers, on aspire davantage à une collaboration dans le travail quotidien (que ce soit sur un plan professionnel ou domestique) quand d'autres agriculteurs se lient avec des conjointes qui seront des partenaires pour la vie économique et domestique du ménage, mais s'investiront très peu, voire pas du tout, dans l'exploitation. Le choix d'une conjointe socialement proche est ainsi crucial lorsque les hommes sont engagés ou vont s'engager dans l'élevage laitier ou la polyculture-élevage. Il l'est beaucoup moins pour les productions céréalières ou pour la vigne. De même, les gros exploitants ont moins besoin de collaboratrices que les petits exploitants ou se réferent à une vision

19 Cette stratégie, définie au sein du couple, n'efface pas pour autant le bénéfice secondaire que peut avoir une conjointe à valoriser personnellement ses capitaux.

${ }_{20}$ Une vérification par un test statistique indique que l'on ne peut trouver de différences significatives en matière d'union avec une conjointe agricultrice (ou encore «agricultrice ou salariée ») entre les exploitants des grandes exploitations et les autres. Rattin conclut également des données du RA2000 que les conjointes travaillent d'autant plus à l'extérieur que les exploitations ont une dimension économique importante (2002b, p. 4).

${ }^{21}$ Et même par des déclarations d'inactivité, tellement l'image que les conjointes ont de leur travail est dévaluée, comme le montre Huet pour le RGP, surtout pour les femmes travaillant dans les exploitations peu spécialisées sur le modèle de «la petite exploitation polyvalente » (Huet, 1981, p. 55). 
Tableau 6. Dimension économique de l'exploitation agricole et profession principale de la conjointe en 2000 et en 1990

\begin{tabular}{lccccc}
\hline \multirow{2}{*}{ Type d'exploitation du chef } & \multicolumn{5}{c}{ Profession de la conjointe } \\
\cline { 2 - 6 } & $\begin{array}{c}\text { Exploitante } \\
\text { agricole }\end{array}$ & $\begin{array}{c}\text { Salariée } \\
\text { agricole }\end{array}$ & $\begin{array}{c}\text { Active non } \\
\text { agricole }\end{array}$ & Inactive & Total \\
\hline Petite exploitation & 41,7 & 3,0 & 31,8 & 23,5 & $100,0(132)$ \\
& 56,0 & 0,8 & 17,8 & 25,4 & $100,0(402)$ \\
Exploitation moyenne & 44,4 & 0,9 & 35,3 & 19,4 & $100,0(232)$ \\
& 55,9 & 0,7 & 21,2 & 22,2 & $100,0(553)$ \\
Grande exploitation & 45,3 & 2,0 & 39,6 & 13,1 & $100,0(717)$ \\
& 56,4 & 0,6 & 21,8 & 21,2 & $100,0(683)$ \\
\hline
\end{tabular}

Source et notes: EE2000, N = 1152 couples où l'homme est agriculteur à titre principal ou secondaire. EE1990, $N=1765$ couples où l'bomme est agriculteur à titre principal ou secondaire.

Lecture : $41,7 \%$ des petits exploitants en 2000 (et $56 \%$ en 1990) ont une conjointe qui est agricultrice de profession.

différente, plus distante, de la place de la femme dans le couple (Rémy, 1976). Les gros agriculteurs, grands céréaliers notamment, viticulteurs de vin de qualité ou certains arboriculteurs, sont plus ouverts aux contacts avec les autres groupes sociaux, ils sont plus proches d'une position de notabilité. Ils sont plus à même de trouver des conjointes qui ont une autre origine sociale et de nouer des alliances avec d'autres groupes sociaux que les petits agriculteurs (du moins ceux d'entre eux qui ne sont pas conduits à s'adjoindre un autre métier et à avoir des contacts avec l'extérieur).

Pour autant, cela ne signifie pas qu'à un autre niveau le travail extérieur des conjointes ne participe pas à une certaine forme de reproduction des positions sociales. Mais pour saisir cela, il faut élargir le point de vue sur le patrimoine transmis par les individus et tenir compte, en plus de la position et de l'origine sociales, d'un autre capital détenu par les individus : le capital scolaire.

\section{L'ouverture et le décentrement de la vie familiale}

Pour comprendre les choix conjugaux des agriculteurs, il faut les rapporter aux transformations en cours dans le processus de reproduction sociale dans notre société, en particulier au poids croissant du capital scolaire. Il faut également les rapporter au nouveau sens que prend la vie conjugale dans ce contexte. L'hétérogamie sociale ne constitue pas en soi un obstacle pour la reproduction du groupe social agricole dans la mesure où l'ancienne appartenance sociale de la conjointe est effacée par la nouvelle. C'est justement ce qui n'a pas lieu aujourd'hui dans l'agriculture : les jeunes femmes en majorité n'épousent plus la profession de leur conjoint mais gardent la leur ${ }^{22}$. Les différents cloisonnements sont autant de distances introduites entre l'agriculteur, son

22 La formule « j'épouse l'agriculteur, pas l'agriculture » est fréquemment exprimée par des femmes, parfois même d'origine agricole, qui ne veulent absolument pas retrouver la situation de dominée au sein du couple et l'engagement physique pénible qu'ont subis leur mère ou belle-mère. 
groupe social et sa conjointe. Nous examinerons dans quelle mesure cette distance peut perturber la pérennité du groupe social des agriculteurs.

\subsection{Le choix du conjoint et le capital scolaire}

Le système d'attribution des positions sociales s'est profondément transformé. La détention d'un titre (sous l'Ancien Régime) ou d'un patrimoine ne commande plus seule cette attribution et le capital scolaire est devenu un critère déterminant pour l'acquisition légitime d'un très grand nombre de positions sociales (Bourdieu, 1989b ; Singly, 1993). Cette transformation a profondément affecté un grand nombre de professions, même si le monde agricole est resté relativement préservé de ces mécanismes: l'activité agricole n'est que partiellement soumise à la possession d'un diplôme et le capital dominant reste le capital économique. Mais des mécanismes qui arriment la profession au système éducatif se sont petit à petit mis en place, mordant sur l'ancienne logique patrimoniale (aides de type DJA - dotation jeune agriculteur conditionnées aux diplômes obtenus), tout en renforçant les positions de la profession établie... et de ses héritiers.

Cette montée du capital scolaire a également profondément modifié le marché matrimonial (Singly, 1987) : la dot scolaire, dont les enfants sont pourvus à la sortie du système éducatif, joue un rôle considérable dans les modalités de rencontre (Bozon et Héran, 2006) et dans le choix du conjoint - en marge des capitaux économiques (capital foncier et capital d'exploitation pour les agriculteurs) que les parents transmettent - et parvient à expliquer certaines unions hétérogames.

Les agriculteurs sont soumis à ces mécanismes : le capital scolaire dont ils sont détenteurs les aide à nouer alliance avec des conjoints socialement mieux dotés qu'eux. Plus le diplôme de l'agriculteur est élevé et moins l'origine sociale de sa conjointe est proche de la sienne ( $c f$. tableau 7). Ce résultat est valable quel que soit l'âge des conjoints masculins.

L'impact du capital scolaire de l'homme est particulièrement visible pour les unions avec des femmes issues des classes moyenne et supérieure, pour lesquelles l'essentiel de ce qui est à transmettre et à conserver réside dans le capital scolaire. En revanche, la possession de capital scolaire ne semble pas favoriser les unions avec des conjointes d'origine ouvrière.

La scolarisation prolongée des agriculteurs les plus jeunes est aussi le signe d'une transformation très importante du milieu agricole : plus la scolarité est longue, plus les contacts sociaux mixtes sont nombreux et les agriculteurs enclins à trouver une conjointe extérieure au monde agricole. De même, les formations techniques constituent des occasions pour le jeune de faire des stages éloignés de l'exploitation familiale et sont autant de lieux de contacts avec des jeunes gens d'autres milieux sociaux et géographiques. L'école obligatoire (formation générale et technique) ${ }^{23}$

23 Il faut ajouter que de plus en plus les familles d'agriculteurs ont des proches appartenant à d'autres groupes sociaux auxquels ils peuvent se comparer, ce qui peut entraîner également une forte distanciation par rapport au monde agricole (Champagne, 2002). 
favorise la multiplication des points de contacts entre milieux sociaux différents. L'école favorise en outre des trajectoires de détour par rapport à la profession agricole. Rattin (1991) montre que les jeunes agriculteurs aujourd'hui ont plus tendance que leurs aînés à prendre une activité salariée avant de s'installer à leur compte en reprenant l'exploitation familiale. Ces détours professionnels représentent des moments importants de distanciation par rapport à l'exploitation familiale, car les individus s'engagent alors dans d'autres rôles et d'autres univers sociaux. Ces détours favorisent donc une certaine individualisation du jeune, c'est-à-dire l'acquisition d'un moi plus distant de l'univers agricole dont il est issu.

Tableau 7. Diplôme des conjoints et origine sociale des conjointes en 2000 et 1990

\begin{tabular}{lcccc}
\hline \multicolumn{1}{c}{ Diplôme des hommes } & $\begin{array}{c}\text { Filles d'origine } \\
\text { agricole }\end{array}$ & $\begin{array}{c}\text { Filles d'origine } \\
\text { ouvrière }\end{array}$ & $\begin{array}{c}\text { Fille d'une autre } \\
\text { origine }\end{array}$ & Total \\
\hline Aucun, CEP ou BEPC & 62,5 & 19,2 & 18,3 & $100,0(360)$ \\
& 75,6 & 10,5 & 11,2 & $100,0(1059)$ \\
CAP, BEP & 55,1 & 23,9 & 21,0 & $100,0(539)$ \\
& 65,7 & 16,1 & 18,2 & $100,0(461)$ \\
Bac ou brevet professionnel & 46,8 & 24,4 & 28,9 & $100,0(156)$ \\
& 40,8 & 15,4 & 43,9 & $100,0(130)$ \\
Diplôme supérieur & 34,2 & 24,1 & 41,8 & $100,0(79)$ \\
& 31,6 & 10,5 & 57,9 & $100,0(38)$ \\
\hline Total & $54,8(622)$ & $22,6(255)$ & $22,6(257)$ & $100,0(1134)$ \\
& $69,2(1169)$ & $13,0(220)$ & $17,7(299)$ & $100,0(1688)$ \\
\hline
\end{tabular}

Source et notes: EE2000, N = 1134 couples où l'homme est agriculteur à titre principal ou secondaire. EE1990, $N=1688$ couples où l'bomme est agriculteur à titre principal ou secondaire.

Lecture: 62,5\% des conjoints qui ont au plus le niveau BEPC en 2000 (et 75,6\% en 1990) ont une conjointe fille d'agriculteur (ou de salarié agricole).

Si l'on résume: la scolarisation conduit nombre de jeunes agriculteurs à une double distanciation par rapport à l'univers agricole. Elle favorise une logique plus personnelle, puisque les jeunes sont porteurs d'un capital valorisé, et la rencontre avec des milieux sociaux assez différents. Cela pousse certains de ces jeunes à tenter des expériences professionnelles qui constituent des détours par rapport à l'installation directe, de même qu'à tenter des expériences amoureuses avec des partenaires venant d'autres origines sociales et appartenant à d'autres mondes professionnels.

\subsection{Le sens de l'alliance et le décentrement de la vie conjugale}

Les agriculteurs ont fonctionné sur un mode plutôt patrimonial. Ce fonctionnement de la famille est aussi très présent dans d'autres catégories sociales où le patrimoine, industriel ou symbolique, est dominant ${ }^{24}$ et contribue à faire des individus avant tout

\footnotetext{
24 Saint-Martin (1993, pp. 111-115) évoque ainsi cette noblesse définie par le nom porté, souvent composée de grands propriétaires terriens qui sont des «héritiers hérités » par le château et les propriétés.
} 
des «membres » d'une famille plutôt que des individus autonomes ${ }^{25}$. Pourtant, la logique individualisante du capital scolaire contribue à transformer la vie conjugale et familiale. Désormais, l'un des buts centraux de la famille est la construction et la validation des identités personnelles (Singly, 1993).

Le modèle de la famille individualiste et relationnelle qui tend à s'imposer surtout dans les classes moyenne et supérieure intellectuelles remplit une double fonction : elle tend à reproduire (voire améliorer) les positions sociales et à construire des individus autonomes par rapport au couple et à la parenté. De ce point de vue, la liberté laissée aux conjointes issues d'autres milieux sociaux d'avoir un emploi en dehors de l'exploitation se comprend comme une manière pour les familles en agriculture de s'adapter au modèle dominant : la conjointe doit aussi disposer de la possibilité réelle (et pas seulement formelle) de valoriser elle-même ses capitaux sur le marché du travail.

Le capital scolaire est un indicateur de la sensibilité à cette logique individualiste dans la famille. Une partie des agriculteurs est désormais plus sensible à la logique qui règne sur le marché matrimonial aujourd'hui et s'appuie désormais sur des relations affectives et sentimentales fortes qui n'excluent ni une certaine distance dans le travail, ni certaines tensions ou conflits entre les deux conjoints (pour le partage des tâches domestiques, pour la décision de travailler à l'extérieur, pour l'éducation des enfants...). La logique de la «centration sur les personnes »(Singly, 1993) est aussi une manière pour les agriculteurs de reconquérir des parts de marché matrimonial au moment même où les règles du jeu donnent un poids de plus en plus important à la psychologisation et à l'amour. C'est donc, si l'on poursuit cette image, à un décentrement de la famille et du couple auquel on assiste dans une large frange du monde agricole, puisque l'exploitation n'est plus le centre exclusif des attentions des individus ${ }^{26}$. Le travail agricole du chef d'exploitation le dimanche nous semble être un bon indicateur de cette « centration » sur l'exploitation.

Le travail à l'extérieur des conjointes est un facteur de réduction du travail des hommes le dimanche (et au-delà le week-end). L'exploitation agricole reste un élément très important de la vie conjugale mais elle ne subordonne pas à sa logique toutes les autres exigences en matière de temps à soi et de temps en couple. L'hétérogamie professionnelle conduit donc à des modifications considérables d'un mode de vie dont Barthez dresse le portrait (1993, p. 475): "L'épouse ayant par ailleurs son métier ne

25 Le monde des ouvriers décrit par Schwartz (2002) manifeste aussi cette résistance à une certaine construction de l'autre en tant que "personne » et non pas «membre » du groupe familial. Ce n'est donc pas le patrimoine seul qui est au cœur de cette forme de construction identitaire, du moins pas le patrimoine économique.

26 Cette plus grande importance de la famille et la relativisation de l'exploitation dans la vie de celle-ci a déjà été analysée, notamment par Mendras (1984, postface), Brun (1989) ou Laurent et Rémy (2000). Insistons sur le fait qu'elle ne se fait pas sans tension, l'agriculteur pouvant avoir plus tendance à défendre l'intégration de la conjointe dans l'exploitation alors que celle-ci peut chercher à préserver son activité personnelle. L'enquête Emploi nous donne peu d'indicateurs sur ces questions. L'enquête ethnographique de Bessière (2006) montre en revanche toutes les tensions qui se manifestent entre conjoints pour la défense d'une activité féminine personnelle et, plus largement, pour le respect de l'autonomie de la scène domestique par rapport à la scène professionnelle agricole. 
participe plus guère à l'activité de son mari. Elle tend plutôt à créer dans la ferme une ouverture sur le monde extérieur, notamment à partir de nouveaux besoins de consommation, en matière d'équipement, de loisir, d'éducation des enfants ». La mise à distance de l'épouse par rapport à l'exploitation, la préservation de son autonomie et de ses appartenances sociales, conduisent à une moindre focalisation du couple sur la vie de l'exploitation.

L'autre partie, plus homogame, du monde agricole reste davantage centrée sur la vie de l'exploitation et sa continuité comme en témoigne la tendance au travail agricole le week-end dans les couples où la conjointe travaille sur l'exploitation. L'ouverture à de nouveaux modes de vie est cependant bien réelle, mais elle reste davantage soumise aux nécessités de l'exploitation.

Tableau 8. Activité professionnelle de la conjointe et travail le dimanche du conjoint

\begin{tabular}{lcccc}
\hline & $\begin{array}{c}\text { Le conjoint travaille } \\
\text { habituellement } \\
\text { le dimanche }\end{array}$ & $\begin{array}{c}\text { Le conjoint travaille } \\
\text { certains dimanches }\end{array}$ & $\begin{array}{c}\text { Le conjoint } \\
\text { ne travaille jamais } \\
\text { le dimanche }\end{array}$ & Total \\
\hline Conjointe agricultrice & 62,3 & 28,0 & 9,7 & 100,0 \\
Conjointe non agricultrice & 43,1 & 43,1 & 13,7 & 100,0 \\
\hline Total & 51,3 & 36,0 & 12,6 & 100,0 \\
\hline
\end{tabular}

Source et note : EE2000, N = 1132 couples où l'homme est agriculteur à titre principal ou secondaire.

Lecture : 62,3\% des exploitants dont la conjointe est agricultrice travaillent habituellement le dimanche.

\subsection{L'hétérogamie et la crise de la succession}

Cette nouvelle situation d'hétérogamie plus courante pose des questions importantes sur le mode de reproduction du groupe agricole. Les enfants des couples hétérogames sont susceptibles d'être dotés à la fois d'un capital culturel "généraliste » approché par le niveau de diplôme de l'enseignement général (que le conjoint masculin possède aussi comme le montre le tableau 7), d'un capital technique agricole et d'un patrimoine foncier. Pour les successeurs, l'importance nouvelle du capital scolaire non agricole a des effets très positifs sur l'activité agricole elle-même: celle-ci réclame en effet toujours plus de compétences générales et nombre d'aides sont délivrées sous condition de diplôme. Mais surtout par rapport à bien d'autres familles, ce capital peut leur ouvrir des destins socioprofessionnels variés. Cela a une conséquence importante: le choix, la décision personnelle devient une dimension centrale de la succession. Celle-ci ne doit plus être vécue comme un destin subi, un échec vécu par les enfants qui n'ont pas pu faire d'études, elle doit être en premier lieu un choix positif, par rapport à d'autres destinées possibles. L'ambiguïté d'un bon nombre de parents agriculteurs à l'égard de l'éventualité de l'installation de leurs enfants (ils seraient contents de ne pas voir leurs enfants continuer un "métier perdu», mais en même temps fiers qu'ils continuent malgré tout) est significative de cette ouverture des destins professionnels (Champagne et Maresca, 1986). Les parents savent en tout cas qu'ils ne peuvent plus guère imposer leurs choix à leurs enfants et que ceux-ci doivent prendre eux-mêmes la décision de s'installer.

En ce sens donc, la succession agricole est plus aléatoire que lorsque la reprise était vécue par beaucoup comme un destin ou faute de mieux pour d'autres (ce qui ne 
manque pas de troubler la profession agricole). Mais cette situation d'une profession agricole choisie par les fils d'agriculteurs est aussi significative des nouvelles manières d'hériter dans notre société. Pour Singly (2003, pp. 27-74), l'héritage aujourd'hui est d'abord construit par les héritiers. Ce sont eux qui ont à choisir ce qu'ils veulent conserver des biens et liens que la génération précédente souhaite transmettre. Bessière (2006) montre à partir d'une étude sur la transmission des exploitations familiales viticoles dans le Cognaçais comment, pour nombre d'agriculteurs aujourd'hui, succéder c'est d'abord choisir sa voie et non pas la subir, c'est d'abord se réapproprier (et parfois transformer, mettre de côté ou détruire) ce qui est transmis par les parents. Les familles hétérogames, particulièrement attentives à l'éducation et à la constitution du capital scolaire de leurs enfants, contribuent par là à cette liberté d'appropriation, à ce choix électif d'une profession agricole. De ce point de vue, c'est dans les couples plus homogames que la succession se construit encore comme un projet familial autant que comme un choix individuel.

Cette plus grande ouverture du champ des possibles a des conséquences importantes : une partie de la profession agricole est plus prête qu'avant à laisser partir ses fils, mais elle est aussi davantage prête à accueillir de nouveaux entrants, comme le montre le recrutement croissant d'agriculteurs sans ascendance agricole. L'absence de successeur familial conduit parfois les parents à choisir des héritiers eux aussi électifs, plutôt que de vendre à un voisin (Barthez, 1999). Une partie de la profession agricole est en train de subir de plein fouet la logique de l'individualisme contemporain (Singly, 2003) et de se transformer profondément. Une autre parât plus attachée aux modes de transmission plus classiques, au risque d'ailleurs de voir des projets s'effondrer quand le successeur désigné ne veut pas reprendre. Cette transformation conduit les agriculteurs du premier segment de l'agriculture à s'efforcer de préserver leur position sociale (quitte à ce que la profession de leurs enfants soit différente de la leur) en valorisant mieux tous les capitaux sociaux (fonciers et culturels) qu'ils détiennent et à faire en sorte que tous les individus de la famille (y compris les conjointes) valorisent au mieux leurs capitaux personnels. L'autre partie de la profession semble plus fonctionner sur un modèle collectif avec la mobilisation de toutes les forces pour défendre l'exploitation et la position de la famille.

\section{Conclusion : L'ouverture conjugale et l'hétérogénéité du monde agricole}

L'amélioration des conditions de vie des agriculteurs et la conquête d'un capital scolaire plus important conduisent à un certain rapprochement avec les autres groupes socioprofessionnels, à une plus grande attractivité à la fois professionnelle et matrimoniale et, par suite, à une plus grande ouverture. L'augmentation du capital scolaire des femmes et des exigences individualistes incite les ménages d'agriculteurs à individualiser leur métier et à cloisonner les mondes professionnels féminin et masculin, alors que naguère les espaces et les tâches étaient affectés aux uns ou aux autres selon le sexe. L'ouverture ne consiste donc pas dans la suppression des distances, des cloisonnements entre groupes socioprofessionnels. La dynamique entre l'évolution des orientations technico-économiques de l'agriculture et l'évolution des origines des 
conjoints est caractéristique et devrait être analysée en détail. Un des motifs de la modernisation de l'agriculture, de la simplification des systèmes de production, voire de la substitution d'un système à un autre, pourrait être à rechercher aussi, en marge des facteurs économiques, agronomiques et politiques, du côté de la moindre disponibilité des femmes en tant que main-d'œuvre familiale. Il s'agit d'ailleurs sans doute moins d'une relation de cause à effet que d'une relation circulaire entre ces deux phénomènes.

L'ouverture ne se fait pas de manière uniforme. Pour une première partie du monde agricole, le cœur de la famille se décentre : le couple se fonde désormais moins qu'avant sur la production et la défense du patrimoine agricole. Cela permet aux agriculteurs de s'adapter au nouveau monde auquel ils sont confrontés. Pour une seconde partie, au contraire, c'est la mobilisation pour l'exploitation et la famille : il s'agit donc d'une plus grande focalisation sur l'exploitation qui reste le cœur de la vie quotidienne. L'ouverture consiste aussi en un élargissement des destinées professionnelles pour les enfants d'agriculteurs. Il s'agit moins d'une reproduction interdite que d'une manière moderne de se reproduire, qui peut passer par une conversion plus facile des divers capitaux possédés par ces familles.

Avec la montée de l'hétérogamie en agriculture, c'est aussi l'unité du groupe socioprofessionnel qui peut être questionnée : si deux ensembles se différencient bien d'un point de vue conjugal (mais aussi familial), ils ont aussi un ancrage très différent dans cet univers, si bien que l'on pourrait parler des mondes de l'agriculture, plutôt que du monde agricole. Pour le premier ensemble, à tendance hétérogame, le décentrement de la vie familiale semble conduire à une certaine distanciation du monde agricole. Pour le second ensemble, à tendance plus homogame et qui insiste sur la mobilisation collective, la proximité au métier et au groupe est clairement réaffirmée. Ces deux ensembles ne se situent pas dans la même position par rapport aux dynamiques démographiques: le premier est en extension puisque les unions hétérogames sont proportionnellement beaucoup plus nombreuses, tandis que le second se réduit entre 1990 et 2000. Dans tous les cas, la tendance croissante à l'ouverture sociale (et pas seulement conjugale) du champ de l'agriculture et ses diverses recompositions (environnementales, en particulier) doivent encourager les statisticiens et les sociologues à éclairer systématiquement l'origine des agriculteurs et agricultrices et de leurs conjoint(e)s dans leurs enquêtes, afin de mieux saisir l'impact des unions hétérogames sur les collectifs de travail, les orientations productives et les itinéraires techniques des exploitations comme sur les stratégies des ménages.

\section{Bibliographie}

Barthez A. (1999) L'installation « hors du cadre familial » et relation adoption, Economie rurale $253,15-20$.

Barthez A. (1993) Une agriculture en mutation, in: La société française. Données sociales, Paris, INSEE, 466-475. 
Barthez A. (1982) Famille, travail et agriculture, Paris, Economica, 192 p.

Barthez A., Laferrère A. (1996) Contrat de mariage et régimes matrimoniaux, Economie et statistique 296-297, 127-145.

Bessière C. (2006) Maintenir une entreprise familiale. Enquête sur les exploitations viticoles de la région délimitée Cognac, Université Paris Descartes, Thèse pour l'obtention du doctorat de sociologie dirigée par Olivier Schwartz, 507 p.

Bessière C. (2004) «Vaut mieux qu'elle travaille à l'extérieur!». Enjeux du travail salarié des femmes d'agriculteurs dans les exploitations familiales, Cabiers $d u$ genre 37, 93-114.

Bourdieu P. (1989a) Reproduction interdite. La dimension symbolique de la domination économique, Etudes rurales 113-114, 15-36.

Bourdieu P. (1989b) La Noblesse d'Etat. Grandes Ecoles et esprit de corps, Paris, Minuit, $569 \mathrm{p}$.

Bourdieu P. (1972) Les stratégies matrimoniales dans le système de reproduction, Annales ESC, numéro spécial « Famille et société », 1105-1127.

Bourdieu P. (1962) Célibat et condition paysanne, Etudes rurales 5-6, 32-136.

Bozon M., Héran F. (2006) La formation du couple, Paris, La découverte, 267 p.

Brun A. (1989) La famille comme unité d'analyse du secteur agricole, Economie rurale 194, 3-8.

Champagne P. (2002) L'béritage refusé. La crise de la reproduction sociale de la paysannerie française 1950-2000, Paris, Seuil, 337 p.

Champagne P., Maresca S. (1986) De la succession familiale à l'installation professionnelle, Paris, INRA, 2 vol., 378 p.

Coulomb P., Delorme H., Hervieu B., Jollivet M. et Lacombe P. (eds) (1990) Les agriculteurs et la politique, Paris, Presses de la FNSP, 594 p.

Delame N., Thomas G. (2007) De plus en plus de conjoints d'agriculteurs travaillent hors de l'exploitation, in: L'agriculture, nouveaux défis, Paris, INSEE, 307 p.

Delphy C. (1998) L'ennemi principal, in: L'ennemi principal. 1. Economie politique du patriarcat, Paris, Syllepse, 31-56.

Delphy C. (1977) Les femmes dans les études de stratification, in: Femme, sexisme et sociétés, Michel A. (dir.), Paris, PUF, 25-39.

Desrosières A. (1978) Marché matrimonial et structure des classes sociales, Actes de la Recherche en Sciences sociales 20/21, 97-107.

Desrosières A., Thévenot L. (1996) Les catégories socio-professionnelles, Paris, La découverte, $127 \mathrm{p}$.

Fiche D., Barthélémy D. et Barthez A. (1993) Fermage, sociétés et famille en agriculture. Résultats de l'enquête sur les structures foncières en 1992, Agreste Données 49, 4 p. 
Girard A. (1980) Le choix du conjoint. Une enquête psycho-sociologique en France, Paris, INEDPUF, $201 \mathrm{p}$.

Huet M. (1981) Les catégories statistiques utilisées pour classer les épouses et les enfants d'agriculteurs : des principes à l'usage effectif, in: Les CSP et leur repérage dans les enquêtes. Etudes méthodologiques, Thévenot L. (dir.), Archives et Documents 38, Paris, INSEE, 41-81.

INSEE (1995) Portrait social : les femmes, Paris, INSEE, 217 p.

Jégouzo G. (1991) L'évolution après 1975 du célibat agricole masculin, Population 1, 41-62.

Kayser B. (1997) La renaissance rurale, Paris, Colin, 316 p.

Lagrave R.-M. (dir.) (1987) Celles de la terre. Agricultrices : l'invention politique d'un métier, Paris, EHESS, 254 p.

Laurent C., Rémy J. (2000) L'exploitation agricole en perspective, Courrier de l'Environnement 41, 5-23.

Mendras H. (1984) [1967] La fin des paysans, Le Paradou, Babel-Actes Sud, 439 p.

Merllié D., Prévot J. (1997) La mobilité sociale, Paris, La découverte, 123 p.

Rattin S. (2006) Les femmes accèdent lentement à des statuts plus avantageux, Agreste Primeur 175, $4 \mathrm{p}$.

Rattin S. (2002a) L'agriculture au féminin se professionnalise, Agreste Cabiers 2, 15-22.

Rattin S. (2002b) Les ménages d'agriculteurs en 2000 : un sur trois est pluriactif, Agreste Cabiers 2, 3-14.

Rattin S. (1992) Moins d'un million d'exploitations agricoles en 1990, Agreste Etudes 15, $68 \mathrm{p}$.

Rattin S. (1991) Profession : jeune agriculteur, Agreste Etudes 7, 35 p.

Rémy J. (1980) Le fantôme du statut de co-exploitante, Pour 73, 38-40.

Rémy J. (1977) Travail, famille et développement agricole : aspects de la vulgarisation féminine, Paris, INRA, 115 p.

Rémy J. (1976) Modèles de formation à l'usage des femmes rurales, Pour 51, 73-79.

Saint-Martin M. de (1993) L'espace de la noblesse, Paris, Métailié, 326 p.

Schwartz O. (2002) Le monde privé des ouvriers, Paris, PUF, 531 p.

Singly F. de (2003) Les uns avec les autres. Quand l'individualisme crée du lien, Paris, Colin, $268 \mathrm{p}$.

Singly F. de (2000) Libres ensemble. L'individualisme dans la vie commune, Paris, Nathan, $253 \mathrm{p}$.

Singly F. de (1993) Sociologie de la famille contemporaine, Paris, Nathan, 128 p.

Singly F. de (1987) Théorie critique de l'homogamie, L'Année sociologique 37, 181-205. 
Vallet L.-A. (1986) Activité professionnelle de la femme mariée et détermination de la position sociale de la famille. Un test empirique : la France entre 1962 et 1982, Revue Française de Sociologie 27(4), 655-696.

Vanderschelden M. (2006) Homogamie socioprofessionnelle et ressemblance en termes de niveau d'études : constat et évolution au fil des cohortes d'unions, Economie et Statistique 398-399, 33-58.

Weber F. (1989) Le travail à côté. Etude d'ethnographie ouvrière, Paris, INRA/EHESS, 210 p. 